\section{A mortalidade por doenças infecciosas no início e no final do século XX no Município de São Paulo}

\section{Deaths due to infectious diseases in the beginning and the end of the $20^{\text {th }}$ century in the City of São Paulo}

\section{Cássia Maria Buchalla}

Departamento de Epidemiologia

Faculdade de Saúde Pública

Universidade de São Paulo

Av. Dr. Arnaldo, 715

01246-904 - Cerqueira César

São Paulo, SP, Brasil

cmbuchal@usp.br

\section{Eliseu Alves Waldman}

Departamento de Epidemiologia

Faculdade de Saúde Pública

Universidade de São Paulo

Ruy Laurenti

Departamento de Epidemiologia

Faculdade de Saúde Pública

Universidade de São Paulo

\section{Resumo}

A melhora das condições de vida do homem durante o século XX contribuiu para transformações da estrutura demográfica e para mudanças dos padrões de morbi-mortalidade. Com o objetivo de discutir essas mudanças, pretende-se comparar os dados de mortalidade do Município de São Paulo em 1901, 1960 e 2000.

As causas de morte foram obtidas das seguintes fontes: Anuários Estatísticos da FSEADE, para 1901; Anuário de 1961 do Departamento de Estatística do Estado e CDROM de Mortalidade do Ministério da Saúde, para o ano de 2000. Os demais dados - de população, de nascidos vivos, coeficientes de mortalidade geral e infantil - foram obtidos do CD-ROM "500 anos de Divisão territorial e 100 anos de Estatísticas Demográficas Municipais" da FSEADE.

Do início até o final do século XX, a população do Município de São Paulo aumentou 36 vezes, o número de nascimentos quase 24 e o número de óbitos 13,6 vezes. No entanto, as taxas de mortalidade diminuíram, a mortalidade geral foi 2,6 vezes menor no ano 2000 e a mortalidade infantil 11,3 vezes menor. A proporção de óbitos por doenças infecciosas declina de $45,7 \%$ do total de óbitos em 1901 para 9,7\% em 2000.

Em 1901, entre as 10 principais causas de morte no Município, 5 eram doenças infecciosas, correspondendo a $37 \%$ das mortes; em 1960, apareciam nesta lista apenas 3 doenças infecciosas (16,1\% dos óbitos), e em 2000 apenas a pneumonia constava entre as principais causas de morte.

Doenças como tétano, difteria, coqueluche, sarampo e escarlatina mostraram-se controladas no final do período. No entanto, a pneumonia, a tuberculose, a septicemia e a aids ainda se situam entre as que merecem especial atenção na área de saúde pública.

Palavras-chave: Mortalidade. Transição epidemiológica. Doenças infecciosas. 
Abstract

The changes in living conditions during the $20^{\text {th }}$ century have contributed to change morbidity and mortality standards, and the population structure. This study aims to present and discuss these changes by comparing mortality data of the City of São Paulo from 1901, 1960 and 2000.

Mortality data were collected from Annual Statistical Reports of Fundação SEADE (State Data Analysis System Foundation), for 1901; from Annual Statistical Reports of the State Department of Statistics, for 1961; and from a CD-ROM of the Ministry of Health, for 2000 .

The population data, number of newborns and infant mortality rates were collected from the CD-ROM "500 anos de Divisão Territorial e 100 Anos de Estatísticas Demográficas Municipais" (500 years of Territory Division and 100 Years of Municipal Demographic Statistics) of FSEADE.

During the 20th century, the population of the City of São Paulo increased 36-fold, the number of newborns 24 -fold and deaths 13.6-fold. On the other hand, mortality rates decreased, general mortality was 2.6 times lower and infant mortality was 11.3 lower in 2000 when compared to 1901 . The proportion of deaths due to infectious diseases decreased from $45.7 \%$ of total deaths in 1901 to $9.7 \%$ in 2000 .

Taking into consideration the 10 main causes of death for 1901, 5 were infectious diseases, corresponding to $37 \%$ of all deaths. In 1960 there were only 3 infectious diseases among the main causes of deaths, accounting for $16.1 \%$ of all deaths, and in 2000 only pneumonia was among the 10 main causes of death.

Tetanus, diphtheria, whooping cough, measles, and scarlet fever were controlled in this period. Nevertheless, infectious diseases as pneumonia, septicemia, tuberculosis, and AIDS still deserve special attention from public health authorities.

Key Words: Mortality. Epidemiologic transition. Infectious diseases.

\section{Introdução}

Até o final do século XIX, as doenças infecciosas e a fome endêmica persistiam entre os principais problemas de saúde pública, sendo responsáveis por elevadas taxas de mortalidade infantil e pela baixa expectativa de vida das populações humanas. Tal situação estava em boa parte relacionada à falta de saneamento, habitações inadequadas, condições de trabalho insalubres e baixo nível de escolaridade, propiciando condições para elevadas taxas de mortalidade por diarréia, varíola, peste, cólera e tuberculose, mesmo em países desenvolvidos. Porém, no decorrer do século XX, a ampliação do saneamento urbano, a melhora nas condições de nutrição, a elevação do grau de escolaridade, o desenvolvimento de novas tecnologias médicas e a ampliação da cobertura dos serviços permitiram uma expressiva melhora nas condições de vida do homem, contribuindo para a acentuada queda na mortalidade por doenças infecciosas, especialmente na infância ${ }^{1}$.

A importante diminuição dos óbitos por doenças infecciosas, em especial pela diarréia, teve influência inegável nas rápidas e significativas transformações da estrutura demográfica e nas mudanças dos padrões de morbi-mortalidade no século XX, caracterizados pelo aumento da expectativa de viva, queda da fecundidade, envelhecimento das populações, diminuição expressiva da mortalidade infantil, aumento da importância das doenças crônico-degenerativas e elevação da morbi-mortalidade decorrente da violência ${ }^{2-4}$.

Com o objetivo de apontar e discutir as mudanças ocorridas nesse período, em especial aquelas verificadas nos padrões decorrentes de mortalidade por doenças infecciosas, pretende-se neste estudo comparar dados de mortalidade por esse grupo de doenças no Município de São Paulo em três momentos: no início, em meados e no final do século XX.

\section{Material e Métodos}

A escolha dos anos de estudos foi baseada na disponibilidade de dados comparáveis. 
Para o ano de 1901, o Anuário Estatístico da FSEADE apresenta a mortalidade para cada tipo de doença. No entanto, para o ano de 1950, apenas o dado bruto - o número de óbitos ou a taxa de mortalidade - é apresentado. Por este motivo, optamos por utilizar dados de 1960, ainda que estes sejam apresentados por grupos de doenças. Os dados de população, número de nascidos vivos, mortes de menores de um ano, coeficientes de mortalidade geral e infantil, foram retirados do CD-ROM "500 anos de Divisão territorial e 100 anos de Estatísticas Demográficas Municipais" da FSEADE. Esta fonte indica, para 1960, dados da população um pouco diferentes daqueles apresentados no Anuário de 1961 do Departamento de Estatística do Estado, de onde foram tirados os dados de mortalidade. Enquanto a FSEADE aponta a população de 1960 para o Município de São Paulo como sendo 3.781.445 habitantes, o anuário apresenta para o mesmo ano 3.851.147 habitantes. Os dados de mortalidade de 2000 foram obtidos das Estatísticas de Mortalidade do Brasil, por meio do CD-ROM.

Para a análise da mortalidade relativas aos anos de 1901, 1960 e 2000 foram selecionadas as dez principais causas e todas as causas infecciosas que formam o capítulo I da Classificação Internacional de Doenças (CID) acrescidas de outras infecções que não estão incluídas no referido capítulo, mas foram consideradas neste estudo. São elas: as meningites, as pneumonias e as broncopneumonias, que são classificadas nos capítulos de doenças do sistema nervoso e do aparelho respiratório, respectivamente, em todas as revisões da CID. Foram ainda consideradas as diarréias, que em 1901 eram classificadas no capítulo de doenças do aparelho digestivo, e o tétano, que na $1^{\text {a }}$ revisão da CID estava no capítulo de doenças do sistema nervoso e dos órgãos dos sentidos. Os dados de mortalidade por doenças infecciosas de 1960 consideraram, além daquelas contidas no $1^{\circ}$ capítulo da CID-7 ${ }^{\text {a }}$ revisão, também a gripe, classificada como doença do aparelho respiratório, e as gastroenterites, classificadas no capítulo de doenças do aparelho digestivo.
Os coeficientes por causa de morte do grupo de doenças infecciosas e parasitárias (capítulo 1 das CID- $1^{\text {a }}, 7^{\text {a }}$ e $10^{\text {a }}$ revisões, somadas às infecciosas classificadas em outros capítulos) foram comparados nos três períodos. Ainda que o ideal fosse padronizar esse coeficiente, isso não foi possível por não existirem nos anuários estatísticos consultados dados da mortalidade por idade e causa nos dois primeiros períodos considerados.

\section{Resultados}

A Tabela 1 apresenta alguns dados demográficos e indicadores de saúde referentes ao Município de São Paulo nos três momentos considerados: 1901, 1960 e 2000. Enquanto a população do Município aumentou 36 vezes, do início até o final do século $\mathrm{XX}$, o número de nascimentos aumentou quase 24 vezes, o número total de óbitos aumentou 13,6 vezes, mas as taxas de mortalidade diminuíram.

A maior diminuição é percebida nas taxas de mortalidade infantil, que diminui 11,3 vezes entre o início e o final do século XX. Já a mortalidade geral foi 2,6 vezes menor em 2000, ao se comparar com a taxa de 1901. A proporção de óbitos de menores de um ano, importante indicador de melhora de qualidade de vida, diminuiu $84,7 \%$.

A proporção de óbitos por doenças infecciosas caiu de $45,7 \%$ do total de óbitos em 1901 para $9,7 \%$ do total de óbitos em 2000 , representando uma queda de $78,8 \%$.

As Tabelas 2, 3 e 4 apresentam as dez principais causas de morte nos anos considerados. Infelizmente, as tabelas de mortalidade por causas, de 1960, apresentam algumas causas agrupadas, como é o caso das neoplasias malignas, que por esse motivo constituem a primeira causa de morte.

Em 1901, os óbitos causados por tumores somavam 72, enquanto em 2000 esse grupo de causas foi responsável por 11.515 óbitos no Município de São Paulo, constituindo o segundo grupo de causas mais freqüentes, superado apenas pelas doenças do aparelho circulatório, com 22.435 óbitos.

O que chama a atenção entre as princi- 
Tabela 1 - Algumas características vitais do Município de São Paulo em 1901 e 1996

Table 1 - Some vital features of the City of São Paulo for 1901 and 1996

\begin{tabular}{lrrr}
\hline & $1901^{\#}$ & $1960^{\$}$ & $2000^{\$}$ \\
\hline População & 286.000 & 3.781 .445 & 10.398 .576 \\
Nascimentos Vivos & 8.763 & 119.775 & 207.462 \\
Óbitos Gerais & 4.993 & 31.361 & 68.090 \\
Mortes em < 1 ano & 1.563 & 7.539 & 3.276 \\
Coeficiente de mortalidade geral $^{*}$ & 17,5 & 8,3 & 6,6 \\
Taxa de mortalidade infantil & 178,4 & 62,9 & 15,8 \\
\% de mortes em < 1 ano & 31,3 & 24,0 & 4,8 \\
\% de mortes por doenças Infec. e Parasitárias** & 45,7 & 22,1 & 9,7 \\
\hline
\end{tabular}

*Taxa por 1.000/Rate per 1,000

**Inclui todas as doenças infecciosas e parasitárias e não somente a Capítulo I da CID-1, CID-7 e CID-10/Includes all infectious and parasitic diseases and not only Chapter I of ICD-1, ICD-7 and ICD-10

" Dados do Anuário Estatístico de São Paulo - 1901 (xerox do SEADE)/Data from São Paulo's Annual Statistical Reports - 1901 (photocopied from SEADE)

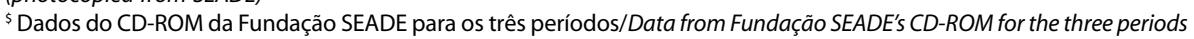

Tabela 2 - Dez principais causas de óbito no Município de São Paulo em 1901

Table 2 - Ten main causes of death in the City of São Paulo in 1901

\begin{tabular}{lcc}
\hline Causas de óbito & No & $\%$ \\
\hline Diarréia e enterite & 1.005 & 20,1 \\
Bronquite aguda & 419 & 8,4 \\
Broncopneumonia/Pneumonia & 346 & 6,9 \\
Moléstias Orgânicas do Coração & 306 & 6,1 \\
Tuberculose pulmonar & 299 & 6,0 \\
Debilidade congênita & 247 & 4,9 \\
Meningite & 121 & 2,4 \\
Congestão e hemorragia cerebral & 85 & 1,7 \\
Malária & 81 & 1,6 \\
Convulsões infantis & 79 & 1,6 \\
\hline Total & 2.988 & 59,84 \\
\hline Total de óbitos (todas as causas) & 4.993 & \\
\hline
\end{tabular}

pais causas de morte é que, em 1901, das dez causas mais importantes 5 eram doenças infecciosas, responsáveis por $37 \%$ do total de óbitos daquele ano. Em 1960, apenas 3 entre as 10 principais causas de morte eram infecciosas: pneumonia, gastroenterites e tuberculose. Estas correspondiam a $16,1 \%$ do total de óbitos daquele ano. Já em 2000, apenas a pneumonia situava-se entre as 10 causas mais freqüentes de morte, como mostra a Tabela 4 . As Tabelas 2, 3 e 4 permitem acompanhar as mudanças no padrão de mortalidade no correr do século XX.
A Tabela 5 apresenta uma lista de doenças infecciosas e parasitárias e as freqüências com que aparecem como causas de morte nos três períodos considerados neste estudo, e os respectivos coeficientes específicos de mortalidade.

Ainda que a lista das doenças consideradas como infecciosas tenha aumentado entre 1901 e 2000, a participação dessas doenças como causa de morte diminuiu muito, como foi comentado anteriormente. Algumas doenças infecciosas, causas freqüentes de morte em 1901, como tétano, difteria, 
Tabela 3 - Dez principais causas de óbito no Município de São Paulo em 1960*

Table 3 - Ten main causes of death in the City of São Paulo in 1960*

\begin{tabular}{lcc}
\hline Causas de óbito* & No & $\%$ \\
\hline Neoplasias malignas (140-205) & 3.789 & 12,1 \\
Doenças arteriais e degenerativas do coração (420-422) & 3.311 & 10,6 \\
Lesões vasculares do SNC (330-334) & 2.418 & 7,7 \\
Pneumonia ( 490- 493) & 2.257 & 7,2 \\
Disenterias (045-048) e Gastroenterites (543, 571 e 572) & 1.935 & 6,2 \\
Outras doenças peculiares à infância e imaturidade & 1.484 & 4,7 \\
sem precisão (780-795) & 1.131 & 3,6 \\
Hipertensão com doença do coração (440-443) & 1.124 & 3,6 \\
Outras doenças do coração (430-434) & 855 & 2,7 \\
Tuberculose do aparelho respiratório (001-008) & 667 & 2,1 \\
Diabetes Mellitus (260) & 18.860 & 60,14 \\
\hline Total & 31.361 & \\
\hline Total de óbitos (todas as causas) & & \\
\hline
\end{tabular}

Fonte: Anuário 1960. Governo do Estado de São Paulo. Departamento de Estatística do Estado, 1961

Source: 1960 Annual Statistical Report. São Paulo State Government. State Statistical Department, 1961

* Por motivos de apresentação dos dados no Anuário de 1960, algumas causas estão agrupadas. Causas segundo a 7a Revisão da Classificação Internacional de Doenças.

* In order to present the data of 1960 Annual Statistical Report, some causes have been grouped. Causes according to the $7^{\text {th }}$ Revision of the International Disease Classification.

Tabela 4 - Dez principais causas de óbito no Município de São Paulo em 2000*

Table 4 - Ten main causes of death in the City of São Paulo in 2000*

\begin{tabular}{lcc}
\hline Causas de óbito & No & $\%$ \\
\hline Infarto agudo do miocárdio (I21) & 6.304 & 9,2 \\
Agressão por disparo de arma de fogo ou não especificada (X95) & 3.627 & 5,3 \\
Pneumonia por microrganismo não especificado (J18) & 3.195 & 4,7 \\
Diabetes Mellitus não especificada (E14) & 2.361 & 3,4 \\
Doença isquêmica crônica do coração (I25) & 2.174 & 3,2 \\
Outras doenças pulmonares obstrutivas crônicas (J44) & 2.077 & 3,0 \\
Acidente Vascular cerebral (I64) & 1.954 & 2,9 \\
Agressão por meios não especificados (Y09) & 1.776 & 2,6 \\
Neoplasia maligna dos brônquios e dos pulmões (C34) & 1.401 & 2,0 \\
Cardiomiopatias (I42) & 1.259 & 1,8 \\
\hline Total & 26.128 & 38,24 \\
\hline Total de óbitos (todas as causas) & 68.326 & \\
\hline
\end{tabular}

Fonte: Ministério da Saúde - estatísticas de mortalidade Brasil. CD-ROM.

Source: Ministry of Health - mortality statistics for Brazil. CD-ROM

coqueluche, escarlatina e sarampo, foram controladas e hoje são bem menos prevalentes.

No entanto, algumas doenças continuam apresentando taxas elevadas de mortalidade, como é o caso das pneumonias, da tuberculose, septicemias, doenças de Chagas e diarréias infecciosas. Estas constituem, junto com a aids, as doenças infecciosas mais importantes como causa de morte. Ainda que os coeficientes de mortalidade específicos por causa tenham diminuído considera- 
Tabela 5 - Mortalidade por Doenças Infecciosas (nº absoluto e coeficiente x 100.000) no Município de São Paulo, 1901, 1960 e 2000

Table 5 - Mortality due to Infectious Diseases (absolute number and rate per 100,000) in the City of São Paulo, 1901, 1960 and 2000

\begin{tabular}{|c|c|c|c|c|c|c|}
\hline \multirow[b]{2}{*}{ Doenças } & \multicolumn{2}{|c|}{1901} & \multicolumn{2}{|c|}{1960} & \multicolumn{2}{|c|}{2000} \\
\hline & $\mathrm{N}^{\circ}$ & Coef. & $\mathrm{N}^{\circ}$ & Coef. & $\mathrm{N}^{\circ}$ & Coef. \\
\hline Cólera & 4 & 1,4 & - & - & - & - \\
\hline Febre Tifóide e Paratifóide & 68 & 23,8 & 2 & 0,05 & - & - \\
\hline Diarréia e gastroenterites ( 1 ) & 1005 & 351,4 & 1935 & 51,2 & 235 & 2,3 \\
\hline Tuberculose Pulmonar & 299 & 104,5 & 855 & 22,6 & 505 & 4,9 \\
\hline Outras Tuberculoses. & 38 & 13,3 & 131 & 3,5 & 80 & 0,7 \\
\hline Hanseníase & 5 & 1,7 & 7 & 0,2 & 3 & 0,02 \\
\hline Tétano (2) & 12 & 4,2 & 150 & 4,0 & - & - \\
\hline Difteria & 5 & 1,7 & 86 & 2,3 & - & - \\
\hline Coqueluche & 60 & 21,0 & 77 & 2,0 & - & - \\
\hline Escarlatina & 2 & 0,7 & 3 & 0,1 & - & - \\
\hline Meningites (3) & 121 & 42,3 & 264 & 7,0 & 86 & 0,8 \\
\hline Septicemias $(4)^{*}$ & 19 & 6,6 & - & - & 307 & 2,9 \\
\hline Erisipela * & 7 & 2,4 & - & - & 28 & 0,3 \\
\hline Sífilis & 31 & 10,8 & 148 & 3,9 & 3 & 0,02 \\
\hline Raiva & - & - & 8 & 0,2 & - & - \\
\hline Encefalites $(5)^{*}$ & 4 & 1,4 & - & - & 5 & 0,05 \\
\hline Febre Amarela* & 3 & 1,0 & - & - & - & - \\
\hline Varíola & 46 & 16,1 & 5 & 0,1 & - & - \\
\hline Sarampo & 57 & 19,9 & 328 & 8,7 & - & - \\
\hline Hepatites Virais (6) & - & - & - & - & 175 & 1,7 \\
\hline AIDS & - & - & - & - & 1379 & 13,3 \\
\hline Malária (7) & 81 & 28,3 & 2 & 0,05 & - & - \\
\hline Leishmaniose* & - & - & - & - & 2 & 0,02 \\
\hline Doença de Chagas $(8)^{*}$ & - & - & - & - & 345 & 3,3 \\
\hline Esquistossomose* & - & - & - & - & 33 & 0,3 \\
\hline Gripe (9) & 13 & 4,5 & 176 & 4,6 & 7 & 0,07 \\
\hline Pneumonias e Broncopneumias (9) & 346 & 121,0 & 2257 & 59,7 & 3234 & 31,1 \\
\hline Poliomielite Aguda & - & - & 154 & 4,1 & - & - \\
\hline TOTAL de Doenças Infecciosas (10) & 2281 & & 6920 & & 6583 & \\
\hline
\end{tabular}

* Doenças não especificadas como causa de morte no Anuário Estatístico do Estado de São Paulo, 1960.

*Diseases not specified as cause of death in the Annual Statistical Report of the State of São Paulo, 1960.

(1) Na CID-1 e na CID-7 essas doenças eram classificadas no Capítulo de Doenças do Aparelho Digestivo/(1) In ICD-1 and ICD-7 these diseases were classified in the Chapter Diseases of the Digestive System

(2) Na CID-1 estava no Capítulo de Doenças do Sistema Nervoso. /(2) In ICD-1, they were in the Chapter of Diseases of the Nervous System

(3) Os dados de 1901 referem-se a todas as meningites, classificadas como doenças do Sistema Nervoso e dos Órgãos dos sentidos. Os dados de 1960 referem à infecções meningocócicas, do capítulo 1, mais as meningites não meningocócicas, classificadas no capítulo de doenças do Sistema Nervoso, na CID7a revisão. /(3) Data from 1901 refers to all meningitis, classified as Diseases of the Nervous System and Organs of Senses. The data from 1960 refers to meningococcal infections from chapter 1, plus non-meningococcal meningitis, classified in the chapter of Diseases of the Nervous System in ICD-7th revision

(4) Na CID-1 aparece como "Infecções Purulentas e Septicemias"/(4) in ICD-1 they are in "Purulent Infections and Septicemia"

(5) Na CID-1 estava no Capítulo de Doenças do Sistema Nervoso. Na CID-10 inclui todas as encefalites/(5) In ICD-1, they were in the Chapter of Diseases of the Nervous System. In ICD-10 all encephalitis are included

(6) Não existia esse diagnóstico na CID-1/(6) This diagnosis did not exist in ICD-1

(7) Na CID-1 aparece como "Febre Intermitente e Caquexia Malárica"/(7) In ICD-1 it is "Intermittent Fever and Cachexia due to Malaria"

(8) Ainda não havia sido descrita em 1901/1902/(8) It had not been described yet in 1901/1902

(9) Na CID-1 era considerada no mesmo Capítulo que as demais doenças infecciosas, nas demais revisões da CID essa doença está no Capítulo de Doenças do Aparelho Respiratório/(9) In ICD-1 it was classified in the same Chapter as other infectious diseases; in the remaining revisions of ICD, the disease is in the Chapter of Diseases of the Respiratory System

(10) Considerando todas as infecções classificadas na CID e não apenas as listadas nesta tabela/(10) Taking into consideration all infections classified in ICD and not only the ones listed in this table 
velmente, essas doenças apresentam coeficientes bastante elevados em 2000, como indica a Tabela 5 .

\section{Discussão}

A análise da tendência secular das doenças permite não só estabelecer hipóteses sobre os fatores envolvidos no processo responsáveis pelas mesmas, mas também avaliar as medidas e as ações de saúde pública, e o reflexo da melhora da qualidade de vida na saúde da população, entre outros fatores.

A Tabela 1 mostra os dados demográficos que traduzem o crescimento da cidade de São Paulo: o aumento em 36 vezes da população e o aumento do número de nascimentos, que em 2000 foi 23 vezes maior do que em 1901. São Paulo teve, na primeira metade do século XX, uma das maiores taxas migratórias do país, em decorrência tanto da migração externa como interna.

Já o coeficiente de mortalidade infantil diminuiu 2,6 vezes no período. Este indicador, um dos mais sensíveis para se avaliar as condições de saúde das populações, não apresentou um declínio contínuo no decorrer do século XX, como indica Antunes (1998). Durante a década de 60 e o início dos anos 70 assistimos o recrudescimento da mortalidade infantil, fato que pode ter contribuído para chegarmos ao final do século com uma mortalidade infantil ainda elevada, especialmente em distritos onde residem os segmentos mais pobres da população ${ }^{5}$.

Em relação à mortalidade geral, que foi aqui colocada apenas como um parâmetro, uma vez que só permite uma análise mais profunda se for padronizada, nota-se que era bastante elevada em 1901. Naquele ano, sem qualquer grande epidemia relatada, $1,7 \%$ da população estimada foi a óbito no Município de São Paulo.

Segundo Antunes (1998), a transição demográfica neste município ocorreu apenas a partir da metade do século passado portanto mais tarde e de forma mais lenta do que nas cidades européias ou norte americanas, apesar das importantes mudanças ocorridas na cidade de São Paulo no início do século XX, como resultado da industrialização e da urbanização ${ }^{6}$.

Nas primeiras décadas de 1900, cerca de $50 \%$ dos óbitos tinham como causa as doenças infecciosas, com cerca de metade deles atribuídos às diarréias, enquanto a tuberculose era responsável por algo em torno de $10 \%$ das mortes. As pneumonias, difteria, sarampo, varíola, coqueluche, febre tifóide e malária, ainda que menos relevantes em termos relativos, destacavam-se por apresentar elevadas taxas de mortalidade, como cita Waldman ${ }^{7}$, e que também pode ser verificado na Tabela 5 .

A mortalidade por doenças infecciosas, grupo importante como causa de morte no início do século passado, era alta em São Paulo e também em regiões já desenvolvidas naquela época. Em 1900, à semelhança do que ocorria em áreas desenvolvidas, como os EUA, no Município de São Paulo 33\% dos óbitos eram devidos à pneumonia, tuberculose e diarréias ou enterites ${ }^{8}$. No final do século XX, temos no Município de São Paulo um padrão de mortalidade muito próximo ao das áreas industrializadas. Enquanto em 1997, nos EUA, as doenças do coração e os cânceres respondiam por $54,7 \%$ das mortes, a pneumonia, a influenza e a aids, por $4,5 \%^{8}$. Em São Paulo, aqueles dois grupos de doenças crônicas foram responsáveis por $49,6 \%$ do total de óbitos de 2000 e apenas a pneumonia por $4,7 \%$, como mostra a Tabela 4 .

A mudança de padrão da mortalidade, caracterizada pelo declínio dos óbitos causados pelas doenças infecciosas e pelo aumento das mortes por doenças crônicas e causas externas, pode ser avaliada através das Tabelas 2, 3 e 4. Em 1901 (Tabela 2), das dez principais causas de morte, 5 eram doenças infecciosas, que respondiam por $45,7 \%$ da mortalidade. Em 1960, embora as doenças fossem apresentadas em grupos, apenas as pneumonias, as gastroenterites e a tuberculose entram no elenco das principais causas de morte. Já no ano 2000, apenas as pneumonias estão na lista das 10 principais causas de morte do Município de São Paulo.

As mudanças no elenco das causas de morte traduzem o resultado das transfor- 
mações ocorridas na sociedade que levaram à diminuição da mortalidade por doenças infecciosas, diminuição da mortalidade infantil e da natalidade, aumento da esperança de vida ao nascer, e envelhecimento da população, entre outros.

Ainda que persista, entre as doenças infecciosas, como importante causa de morte até hoje, a queda da morbi-mortalidade por diarréia foi o que determinou maior impacto nos indicadores de saúde do Município de São Paulo, sendo a principal responsável pela acentuada queda da mortalidade em menores de 5 anos, observada nos três momentos do século XX estudados. Este fato repercutiu na estrutura demográfica do município, contribuindo para o aumento da esperança de vida e, portanto, com a tendência de envelhecimento da população ${ }^{6}$.

Fatores socioeconômicos são apontados na literatura como determinantes da queda da mortalidade infantil. Entre esses, o nível de escolaridade da mãe, a disponibilidade de água tratada e de coleta de esgoto para grande parte da população, e a adoção de medidas ou cuidados básicos de higiene e de nutrição $0^{9,10}$.

As doenças passíveis de prevenção por vacinação, que no início do século eram relevantes em seu conjunto, praticamente desapareceram como causa de óbito a partir da segunda metade dos anos 80. A importância da proteção conferida pelas vacinas pode ser avaliada pelos dados apresentados neste estudo, comparando a mortalidade por esse grupo de doenças no início e no final do século, e também salientando o fato de, apenas em relação ao sarampo, a ampla vacinação infantil contra essa doença ter evitado um milhão de mortes no mundo, por ano, na última década do século $\mathrm{XX}^{11}$.

No entanto, apesar das mudanças ocorridas na sociedade e no padrão de comportamento das doenças, algumas doenças infecciosas mantêm sua relevância em saúde pública. A tuberculose, importante causa de óbito no início do século passado, ainda se destaca entre as mais expressivas, tendo inclusive, apresentado tendência de aumento no período de 1986 a 1996. Os níveis eleva- dos de mortalidade por essa doença não são aceitáveis em face da existência de tratamento eficaz, da boa cobertura de serviços de saúde no município e do acesso universal e gratuito ao diagnóstico e tratamento; por outro lado, não são coerentes com a evolução dos indicadores de saúde e com a tendência da morbi-mortalidade pelas demais doenças infecciosas. Isso se deve, provavelmente, ao fato de ser uma moléstia infecciosa de evolução longa, de tratamento prolongado e cujo controle está intimamente ligado ao desempenho dos serviços de saúde, no sentido de garantir a adesão do paciente ao tratamento, além da mobilidade para se deslocar-se até o domicílio e investigar os comunicantes. É verdade que também sofreu o impacto da introdução da aids, sendo a co-infecção HIV/tuberculose uma das principais causas de recrudescimento da morbi-mortalidade por essa causa, em vários países, inclusive os industrializados ${ }^{12}$.

Outra doença respiratória que, à semelhança da tuberculose, se mantém com elevadas taxas de mortalidade é a pneumonia. Atualmente, entre as doenças infecciosas, é a principal causa de óbito no Município de São Paulo, e continua sendo importante também em países desenvolvidos, como mostra trabalho recentemente publicado, onde a pneumonia e a influenza estão entre as dez principais causas de morte nos EUA em $1997^{\circ}$.

Entre as doenças emergentes no século XX, sem dúvida a gripe, com a grande pandemia de 1918, e a aids são as que mais óbitos causaram. A primeira foi responsável pela inversão da queda da mortalidade geral no Brasil, que chegou a um coeficiente de 28,8 por 1000 habitantes em $1918^{6}$. Já a aids, desde seu aparecimento, no início da década de 80 , até os dias atuais, continua sendo um importante problema de saúde pública nacional, e vale a pena relatar que a cidade de São Paulo é responsável por $20 \%$ do total de casos de aids do país, apresentando em 2000 um coeficiente de mortalidade de 26,9 por 100.000 habitantes $^{13}$.

$\mathrm{O}$ fato de o século XX ter sido o período de maior avanço tecnológico, da democratização de bens, serviços e de informação, 
torna a análise da mortalidade um motivo de reflexão.

As transformações sociais e econômicas ocorridas após a segunda guerra mundial, assim como o rápido desenvolvimento da ciência e da tecnologia, influenciaram, em boa parte do globo, mudanças expressivas no estilo de vida e nas relações entre pessoas e nações, induzindo, por conseqüência, alterações significativas no próprio perfil das doenças infecciosas, que passaram a ser condicionadas por um conjunto muito mais complexo de fatores ${ }^{14}$. Tais fatores estão intimamente relacionados aos modelos de desenvolvimento econômico, às políticas de industrialização e às suas principais conseqüências, entre elas: o rápido processo de urbanização, as alterações ambientais nas cidades e no campo, as migrações e o aumento do intercâmbio internacional, que assumiu o papel de "vetor cultural" na disseminação das doenças infecciosas.

Desses modelos de desenvolvimento econômico decorrem também as formas de incorporação das novas tecnologias médicas, a ampliação do consumo de alimentos industrializados e, em vários países, a desestruturação dos serviços de saúde pública $^{15-17}$. Essa nova realidade acelerou o processo de disseminação de microrganismos e parasitas e modificou sua interação com o homem, de forma mais rápida do que anteriormente, criando condições para a emergência e difusão de novas doenças infecciosas, para a modificação do comportamento de doenças já conhecidas e para o ressurgimento de doenças consideradas, por equívoco, erradicadas $^{18,19}$.

O monitoramento da incidência dessas doenças, juntamente com o conhecimento das alterações sociais e ambientais, pode permitir a elaboração de planos e estratégias de controle, assim como a implantação de medidas que possam reverter esse quadro e diminuir o risco de morte por esse grupo de doenças preveníveis.

\section{Referências}

1. Beaglehole R, Bonita R. Public health at the crossroads. Cambridge: Cambridge University Press; 1997.

2. Bobadilha JL, Frenk J, Lozano R. Freijka T, Stern C. The epidemiologic transition and health priorities. In: Jamison DT. Diseases control priorities in developing countries. Oxford Medical Publications: Oxford University Press; 1993, p. 161-87.

3. Frenk J, Bobadilla JL, Sepulveda J, Cervantes ML. Health transition in middle-income countries: new challenges for health care. Health Policy And Planning 1989; 4(1): 29-39.

4. Omran AR. The Epidemiologic Transition: A theory of the epidemiology of population change. Milbank $Q$ 1971; 49: 38-74.

5. Monteiro CA, Freitas ICM. Evolução de condicionantes socioeconômicos da saúde na infância na cidade de São Paulo (1984-1996). Rev Saúde Pública 2000; $34(6$ Supl): 8-12.

6. Antunes JLF. "Grow and multiply": social development, birth rates and demographic transition in the Municipality of São Paulo, Brazil, time-series for 1901-1994. Rev Bras Epidemiol 1998; 1(1): 61-78.
7. Waldman EA. Doenças infecciosas: A herança para o século XXI. Médicos 1998; 1(5): 94-8.

8. Centers for Disease Control and Prevention. Achievements in public health, 1900-1999: control of infectious diseases. MMWR 1999; 48(29): 621-9.

9. Murray JL, Chen LC. In search of a contemporary theory for understanding mortality change. Soc Sci Med 1993; 36: 143-55.

10. Powles J. Changes in disease patterns and related social trends. Soc Sci Med 1992; 35: 377-87.

11. Waldman EA. A transição epidemiológica : tendências e diferenciais dos padrões de morbimortalidade em diferentes regiões do mundo. $O$ mundo da saúde 2000; 24(24): $10-8$.

12. Elender F, Bentham G, Langford I. Tuberculosis mortality in England and Wales during 1982-1992: Its association with poverty, ethnicity and AIDS. Soc Sci Med 1998; 46: 673-81.

13. Ministério da Saúde. Coordenação Nacional de DST/ AIDS. Boletim ano XV, no 2, outubro de 2001 a março de 2002. 
14. Waldman EA. Doenças Infecciosas Emergentes e Reemergentes. Revista da USP 2001; 51: 128-127.

15. Altekruse SF, Cohen ML, Swerdlow DL. Emerging foodborne diseases. Emerg Infect Dis 1997; 3(3): 28593.

16. Fidler JD. Globalization, international law, and emerging infectious diseases. Emerg Infec Dis 1996; 2(2): 77-84.
17. Morse SS, Hughes JM. Developing and integrates epidemiologic approach to emerging infectious diseases. Epidemiol Rev 1996; 18(1): 1-3.

18. Morse SS. Factors in the emergence of infectious diseases. Emerg Infect Dis 1995; 1(1): 7-15.

19. Centers for Disease Control and Prevention. Addressing emerging infectious disease threats. A prevention strategies for the United States, 1994.

Recebido em: 10/08/2000 Versão final reapresentada em: 04/04/2003

Aprovação em: 16/09/2003 